\title{
Ist die Aristolochiasäure in der Asarum-Pflanze tatsächlich kanzerogen? Die wichtige Bedeutung epidemiologischer Effektmodifikation - mit einem klinischen Fallbeispiel
}

\author{
Johannes G. Schmidt ${ }^{a}$ Severin Bühlmann ${ }^{b} \quad$ Ying Shao $^{c}$ \\ astiftung Paracelsus heute und Praxiszentrum Meinradsberg, Einsiedeln, \\ bPhytax-Labor, Schlieren, \\ cPraxiszentrum Meinradsberg, Einsiedeln, Schweiz
}

\section{Schlüsselwörter \\ Aristolochiasäure · Chinesische Kräuternephropathie · \\ Kanzerogenität · Kausalität · Effektmodifikation · Altchinesische \\ Medizin · Traditionelle Chinesische Medizin, TCM · Nutzen}

\section{Zusammenfassung}

Die Verwendung von Xi Xin (Asarum) ist 2010 verboten worden, weil es Spuren der als kanzerogen betrachteten Aristolochiasäure enthält. Nach den in der Klinischen Epidemiologie üblichen Kausalitätskriterien lässt sich eine Kanzerogenität von Aristolochia-Pflanzen jedoch nicht belegen; als «Beweis» liegen nur die Ergebnisse von Tierversuchen vor, die irreführend sein können. Aristolochia-Pflanzen wie Guang Fang Ji (Aristolochia fangchi) sind in der chinesischen Medizin seit alters her der kalten Wirkung wegen mit Vorsicht verwendet worden, ebenso wie das erst in jüngerer Zeit eingesetzte Guan Mu Tong (Aristolochia manshuriensis). Kalte Arzneien schwächen das Nieren-Yang. Im Fall der ominösen belgischen Nephropathiefälle erfolgte eine mehrfach kontraindizierte Verwendung dieser chinesischen Mittel. Xi Xin hingegen enthält nur wenig Aristolochiasäure, wirkt warm und schwächt die Niere nicht. Es fällt auf, dass das Asarum-Verbot mit vielen Medikamenten der Pharmaindustrie kontrastiert, deren kanzerogene Wirkungen aufgrund kontrollierter Studien tatsächlich gut dokumentiert sind und die dennoch zugelassen sind. Sinnvollerweise, denn wir erwarten vom Arzt, dass er Medikamente dort richtig anwendet, wo sie mehr nützen als schaden. Entscheidend sind also Effektmodifikationen, die in einem zu praxisfremden, modernen Sicherheitsverständnis vergessen zu gehen drohen. Das Asarum-Verbot in der Schweiz entspricht einer durch den «Volkswillen» obsolet gewordenen Benachteiligung komplementärmedizinischer Arzneien gegenüber den Arzneien der Pharmaindustrie.

\author{
Keywords \\ Aristolochic acid - Chinese herb nephropathy - Carcinogenicity . \\ Causality - Effect modification - Ancient Chinese medicine . \\ Traditional Chinese Medicine, TCM $\cdot$ Clinical benefit
}

\section{Summary \\ Is the Carcinogenic Effect of Aristolochic Acid in Asarum Plants Well- Established? The Important Role of Effect Modification in Epidemiologic Analysis - Including a Clinical Case Study}

The use of Xi Xin (Asarum) has been prohibited in 2010 because of its small amount of aristolochic acid which is said to be carcinogenic. Following the causality criteria used in clinical epidemiology, however, the carcinogenic effect of Aristolochia plants appears rather unlikely. The proof of the supposed carcinogenicity is based merely on animal experiments which may be misleading. From ancient times, Aristolochia plants such as Guang Fang Ji (Aristolochia fangchi) as well as the later used Guan Mu Tong (Aristolochia manshuriensis) were known to be applied with caution because of their cold property. And cold medicines may impair the kidney Yang. In the dubious cases of the socalled Chinese herb nephropathy in Belgium, these Chinese medicines have been used improperly in multiple respects. Xi Xin, however, contains very small amounts of Aristolochic acid, has a warming property and has never been involved in kidney problems. The prohibition of Asarum is in striking contrast to the continuous tolerance of many drugs of the conventional pharmaceutical industry for which a carcinogenic effect is well-established. With good reason, as we expect doctors to administer drugs properly in those cases in which the benefit outweighs the risks. Thus, effect modifications are important and must not be forgotten in the modern zeal for absolute safety. In Switzerland, the prohibition of Asarum represents a discrimination of complementary medicines versus the drugs of the pharmaceutical industry, and this is an obsolete contradiction to the 'Will of the People.'

$\begin{array}{ll}\text { KARGER } & \begin{array}{l}\text { @ 2012 S. Karger GmbH, Freiburg } \\ 1015-0684 / 12 / 0244-0242 \$ 38.00 / 0\end{array} \\ \begin{array}{l}\text { Fax }+497614520714 \\ \text { Information@Karger.de } \\ \text { www.karger.com }\end{array} & \begin{array}{l}\text { Accessible online at: } \\ \text { www.karger.com/szg }\end{array}\end{array}$

Dr. med. Johannes G. Schmidt

Stiftung Paracelsus heute

Praxiszentrum Meinradsberg

Ilgenweidstrasse 3, 8840 Einsiedeln, Schweiz

Tel. +41 55-4188191, Fax -4188192

schmidt@paracelsus-heute.ch 


\section{Mots-clés}

Acide aristolochique - Néphropathie aux herbes chinoises .

Cancérogénicité · Causalité · Modification de l'effet · Ancienne

médecine chinoise $\cdot$ Médecine traditionnelle chinoise, MTC · Bénéfices

\section{Résumé}

L'acide aristolochique que contient la plante appelée «asaret» est-il bel et bien cancérigène? L'importance déterminante de la modification de l'effet sur le plan épidémiologique illustrée par un exemple clinique Il est interdit d'utiliser la plante Xi Xin (Asarum) depuis 2010, car celle-ci contient des traces d'acide aristolochique, substance considérée cancérigène. Selon les critères de causalité habituels de l'épidémiologie clinique, il est toutefois impossible de prouver la cancérogénicité de l'acide aristolochique. Les résultats des études sur l'animal en constituent l'unique «preuve», et ceux-ci pourraient être trompeurs. En médecine chinoise, les plantes aristoloches comme le Guang Fang Ji (Aristolochia fangchi) sont traditionnellement utilisées avec précaution en raison de leur effet refroidissant. C'est également le cas pour le Guan Mu Tong (Aristolochia manshuriensis), dont l'emploi est plus récent. Les médicaments refroidissants affaiblissent le Yang des reins. Dans le cas des nombreuses néphropathies recensées en Belgique, on a eu recours à ce remède chinois sans prendre en compte ses nombreuses contre-indications. Le Xi Xin ne contient pourtant qu'une faible quantité d'acide aristolochique. II possède de plus un effet réchauffant et n'affaiblit pas les reins. II est frappant de constater l'interdiction de l'asaret quand de nombreux médicaments de l'industrie pharmaceutique, dont les effets cancérigènes ont été bien documentés dans diverses études contrôlées, restent autorisés. On peut s'attendre de manière raisonnable à ce que le médecin choisisse un médicament présentant un rapport bénéfice/risquefavorable.En outre, lesmodifications de l'effet constituent un aspect déterminant, mais risquent cependant d'être occultées par la médecine moderne, trop éloignée de la réalité dans son discernement de l'innocuité d'un médicament. L'interdiction de l'asaret en Suisse reflète l'image obsolète qu'a le grand public des produits de la médecine complémentaire par rapport aux médicaments de l'industrie pharmaceutique.

\section{Einführung}

Alles ist Gift oder Heilmittel - es kommt darauf an! Keiner beschreibt nach rechtem Grund, was in den Dingen der Natur sei. Alle schreiben sie nur nach dem Hörensagen! (Paracelsus)

Swissmedic hat am 2. Februar 2010 die Verwendung von Xi Xin (Asarum), eine Arznei der chinesischen Medizin, verboten, da sie Spuren der als kanzerogen betrachteten Aristolochiasäure enthält. Vorausgegangen war ein Verbot in EU-Ländern. Auffällig scheint vielleicht, dass dieses in gewissen Fällen sehr nützliche Mittel der Komplementärmedizin ganz verboten wird, wo wir doch in der konventionellen Pharmakotherapie zahllose Mittel verwenden, die kanzerogen sind [1]. Es geht in der Medizin vernünftigerweise immer darum, Nutzen und Nebenwirkungen in einem Gleichgewicht zu halten, bei dem der Nutzen überwiegt. Ein Verbot aller Substanzen, die potenziell kanzerogen sind, käme deshalb keinem vernünftigen Menschen in den Sinn.

Wir wollen hier der Frage nachgehen, inwieweit eine Kanzerogenität von $X i$ Xin überhaupt dokumentiert ist. Auch die in der chinesischen Medizin sehr wichtige Frage der Wirtsinteraktion muss diskutiert werden (im Grunde ist diese Frage in jeder guten Medizin von Bedeutung). Die chinesische Medizin behandelt nicht in erster Linie die Krankheit, sondern den Wirt und seine Konstitution, und sie macht in jedem einzelnen Fall eine Diagnose der Leistungsfähigkeit und der Resistenz der Organe bzw. Funktionskreise. So bestimmt der Wirtszustand die Wahl der Arzneien. Entsprechend wird Xi Xin dann verwendet, wenn der Wirt es ohne Schaden verträgt. Nur jemand ohne praktische Erfahrung würde das Feuer als zu gefährlich verbieten, der nicht weiss, wie damit umzugehen ist und den Nutzen nicht kennt: Alles ist Gift oder Heilmittel, auf die Gegebenheiten kommt es an!

\section{Kanzerogen - was ist Kausalität?}

Kanzerogen ist eine Substanz, welche die Entstehung von Krebs begünstigt oder auslöst. Doch wie weiss man das? Immer wieder werden die Ergebnisse von Tierversuchen herangezogen: Stoffe, die - bei Mäusen und Ratten appliziert oder implantiert - Neoplasien auslösen, gelten als «kanzerogen» - oder «potenziell kanzerogen». Jedoch haben beispielsweise gewisse chirurgische Biomaterialien, die millionenfach implantiert worden sind (Netze bei Hernienoperation), in der Praxis nie zu Sarkombildungen geführt, obwohl sie im Tierversuch bei Ratten und Mäusen Weichteilsarkome auslösen [2]. Ebenso lassen sich Beispiele finden, die das Umgekehrte zeigen. Aus diesem Grund ist es nötig, auf epidemiologische Beobachtungen beim Menschen abzustützen, um eine echte Kanzerogenität zu bestimmen. Die Ergebnisse von Tierversuchen können irreführen und sind deshalb sekundär, wenn nicht wahrscheinlich sogar meist überflüssig.

Der Epidemiologe Rothman beschreibt in seiner Einführung in die Methoden der Epidemiologie die Philosophie des Wissens, wie sie in der Klinischen Epidemiologie mit Bezug auf Karl Popper diskutiert wird [3]. So wird in der statistischen Analyse von Studienresultaten jeweils formal die Nullhypothese widerlegt, um einen Unterschied $\mathrm{zu}$ beweisen. Und dies geht einher mit Poppers Notion, wonach die blosse Bestätigung einer Hypothese durch passende Beobachtungen mit zu viel Irrtumsmöglichkeiten behaftet ist. Zum Beispiel macht Wasser nass und Feuer trocken. Viele Beobachtungen bestätigen dies und 
Tab. 1. Kausalitätskriterien von Hill [3]
1. Stärke des Zusammenhangs
2. Konsistenz
3. Spezifität
4. Zeitliche Abfolge
5. Dosis-Gradient
6. Plausibilität
7. Kohärenz
8. Durch kontrollierte Studien belegt
9. Analogie

scheinen zu beweisen, das Wasser nass macht. Aber auch Feuer macht nass, denn nur mit Feuer entsteht Dampf, der etwa eine Küche nicht am Boden, sondern an Wand und Decke nass machen kann. Im praktischen Kontext braucht es hier das Feuer (oder Druck usw.), um oben nass zu machen. Gibt man andererseits wieder kaltes Wasser in die Pfannen, dann hört der nässende Dampf auf und das (kühlende) Wasser macht dann trocken. Und ein Leck kann je nach Kontext nass machen und so weiter. Eine Hypothesentestung muss durch die Nullhypothesendisziplin also genau bedacht werden, und die externe Validität eines «Beweises» ist auf die jeweiligen Beobachtungsumstände, auf den genauen Kontext, begrenzt. Poppers Philosophie zeigt die Grenzen und Irrtumsmöglichkeiten von Schulwissen, dem über den beschränkten Entstehungskontext hinaus Gültigkeit beigemessen werden will. Ist die Wahrnehmung nicht gestört von bisherigem «Wissen», können die Dinge (besser) gesehen werden, wie sie sind. (Auch das Zen, christliche Mystiker und viele andere machen darauf aufmerksam, dass Menschen dazu neigen, ihre Vorstellungen und ihr «Wissen» auf Dinge zu übertragen, statt die Dinge unverfälscht immer wieder neu zu sehen.)

Wie ebenfalls in Rothmans Einführung in die Epidemiologie zu finden ist, werden in der Klinischen Epidemiologie die Kriterien von Hill gebraucht, um einen Kausalzusammenhang zu etablieren (Tab. 1) [3].

\section{Fehlender Kausalitätsnachweis}

Der mögliche Zusammenhang zwischen Aristolochiasäure und Urothelkarzinomen hat vor 10-12 Jahren Aufsehen erregt $[4,5]$. Für diesen Zusammenhang existieren folgende Zahlen: In der ominösen belgischen Schlankheitsklinik, die Aristolochiasäure-haltige Schlankheitspillen zum Abnehmen verkaufte, zeigte sich bei insgesamt 1700 behandelten Patienten bei Arzt A in $48 \%$ der Fälle eine Nephropathie, bei Arzt B nur in 1\% der Fälle [6]. In Frankreich wurden 2000 Fälle identifiziert, die die Schlankheitspillen eingenommen hatten; eine Nephropathie trat dort in 2 Fällen (1\%o) auf [6]. Bei all diesen Fällen wurden unter anderem die chinesischen Mittel Guang FangJi (Aristolochia fangchi) und Guan Mu Tong (Aristolochia manshuriensis) verwendet. Über Nephropathien bzw. Urothelneoplasien unter Xi Xin, das 1000-mal geringere Mengen an Aristolochiasäure enthält [7], gibt es hingegen keine Berichte. In der chinesischen Pharmakopöe kommt Xi Xin 马兒铃 科植物 als (i) Asarum heterotropoides Fr. Schmidt var. mandshuricum (Maxim) Kitag. 北细辛, (ii) Asarum sieboldii Miq. var. seoulense Nakai 汉城细辛 und (iii) Asarum sieboldii Miq. 华细辛的干燥根和根菱 vor. Im alten China galt nur der Wurzelteil als Arznei, weil die oberen Teile den Alten noch als ungeeignet bekannt waren. Stängel und Blätter enthalten mehr Aristolochiasäure. Nach 1950 wurde in China aus Kostengründen die ganze Pflanze verwendet [8]. Im Praxiszentrum Meinradsberg haben wir seit 1998 hundertfach Xi Xin verwendet, aber nie eine Niereninsuffizienz oder gar ein Urothelkarzinom gesehen. Die Firma Complemedis hat in den letzten 5 Jahren über 2000 Rezepturaufträge mit insgesamt rund $64 \mathrm{~kg} X i$ Xin ausgeführt. Xi Xin wird in der Schweiz seit 15 Jahren verwendet, seit der Gründung dieser Firma (unter dem damaligen Namen Sinecura). Auch im alten und modernen China sind Nephropathien im Zusammenhang mit Xi Xin nie ein Thema gewesen [9].

Der Zusammenhang zwischen Urothelkarzinomen und Aristolochiasäure scheint aufgrund der Fälle von Arzt A ausserordentlich stark zu sein, doch der Zusammenhang ist weit schwächer bei Arzt B und in Frankreich. Und für Xi Xin besteht gar kein Zusammenhang, denn es sind keine Karzinomfälle bekannt. Die Ergebnisse bezüglich der nephropathischen Folgen von Aristolochiasäure sind jedenfalls sehr inkonsistent, sodass sich nicht der Schluss ziehen lässt, Aristolochiasäure sei als (alleinige) Ursache der Urothelkarzinome bewiesen. Immerhin ist in der chinesischen Medizin von alters her bekannt, dass Guang Fang Jikalt wirkt und mit Vorsicht eingesetzt werden muss, ebenso wie das erst in jüngerer Zeit verwendete Guan $M u$ Tong. Kalte Arzneien können bei ungeeignetem und zu langem Gebrauch sowie bei zu hoher Dosierung das Nieren-Yang schädigen. Xi Xin wirkt hingegen warm. Bei Übergewichtigen ist fast ausnahmslos eine schwache und kalte Mitte/Milz als Ursache vorhanden - und im Hintergrund meist auch eine schwache und kalte Niere als Mitursache (Yang-Mangel). Eine Kontraindikation für Guan Mu Tong und Guang Fang Ji.

Die sehr grossen Unterschiede in der Nephropathieprävalenz unter der Schlankheitspille zwischen den beiden belgischen Ärzten und zwischen Belgien und Frankreich machen es unwahrscheinlich, dass der gleiche Schlankheitscocktail allein und speziell die darin enthaltene Aristolochiasäure die Ursache für die Nephropathien sind. $\mathrm{Ob}$ spezifisch Aristolochiasäure oder doch etwas ganz anderes für die beobachteten Nephropathien verantwortlich war, ist aufgrund der Datenlage nicht klar. Klar ist hingegen, dass ein unbekannter (oder bisher nicht veröffentlichter) Faktor für die grossen Unterschiede verant- 
wortlich sein muss. Die in den Schlankheitspillen gleichzeitig enthaltenen Appetitzügler jedenfalls «stellen den Appetit kalt», was deren kältende Nebenwirkungen zeigen: Verstopfung (Peristaltik wird gekühlt), Mundtrockenheit (die Kälte verhindert das Dampfen), Kopfschmerzen (das erdrückte Yang mag nicht mehr bis oben klären), Schlafstörungen (kalt schläft man nicht gut), Blutdruckerhöhungen (Suppression des Nieren-Yang), Nephritiden (falsche Hitze) und Harnverhalten («Einfrieren»). Appetitzügler und das kalte Guan Mu Tong oder Guang Fang Ji zusammen sind eine mehrfach falsche und kontraindizierte Therapie bei der Yang-Schwäche der Übergewichtigen, und eine Potenzierung von Nebenwirkungen mit gefährlichen Folgen kommt nicht unerwartet.

Dass die Aristolochiasäure mutagen und stark kanzerogen wirke, ist letztlich also nur eine Schlussfolgerung aus Tierversuchen $[10,11]$, und Tierversuche können irreführend sein. Für das unentbehrliche und deshalb sehr häufig verwendete $X i$ Xin sind beim Menschen weder Krebsfälle noch eine kausale Rolle dieser Arznei in der Krebsentstehung dokumentiert, sogar mit der fälschlichen Verwendung der ganzen Pflanze statt nur der Wurzel. Somit ist kaum eines der klinisch-epidemiologischen Kausalitätskriterien für Aristolochiasäure als Krebsursache erfüllt - auch nicht für Guan Mu Tong und Guang Fang Ji mit ihrem relativ hohen Gehalt. Auch letztere Spezies wurde über Jahrhunderte verwendet, allerdings mit einer Diagnose und Indikation nach der chinesischen Medizin. Damit ist eine mögliche Kanzerogenität von Aristolochiasäure in diesen Pflanzen (bei falschem Gebrauch) nicht gänzlich ausgeschlossen, aber die Datenlage für die Risiken bei richtigem Gebrauch ist nicht sehr beunruhigend. Für Xi Xin ist die Datenlage sogar sehr beruhigend.

Effektmodifikation - macht ein Heilmittel zum Gift und Gift zu einem Heilmittel

Die Berücksichtigung von Effektmodifikationen ist entscheidend und für eine effektive medizinische Praxis unabdingbar. So sind Guang Fang Ji und Guan Mu Tong wie gesagt ausgesprochen kalte Drogen, die etwa bei Kälte-Nässe-Pathologien kontraindiziert sind. Sie kälten die Niere und sollten bei Yang-Schwäche nur vorsichtig, durch Yang-Arzneien ausbalanciert, oder gar nicht verwendet werden. Übergewicht ist praktisch immer ein YangMangelzustand, denn das Yang fehlt, um die Nahrung gut $\mathrm{zu}$ «verbrennen» und Nässe und Kälte zu «verdampfen», die sich in der Folge als Fettgewebe anlagern. Schlankheitsdrogen sind eine falsche Therapie, die dem zeitgeistigen Wunsch nach schnellen Wundern entspricht, aber nicht der althergebrachten chinesischen Medizin, die immer das Yang zu bewahren trachtet und die Grundursache behan- deln will. Und eine wirkliche Behebung des gesundheitlichen Problems mit günstigen Langzeitfolgen ist ja bei jeder Therapie (auch mit westlichen Medikamenten) zu fordern. Wird also das schon schwache Yang (bei Übergewicht) durch Appetitzügler und falsche «Zauberdrogen» dermassen ruiniert, dann kann die Niere (oder das Blut usw.) ruiniert werden - mit oder ohne Aristolochiasäure. Die bekannten epidemiologischen Daten aus Belgien und Frankreich zeigen eindeutig, dass starke Effektmodifikationen im Spiel sind, die weit stärker sind (500-facher Unterschied in den Nephropathieprävalenzen) als der weit weniger signifikante Zusammenhang zwischen Aristolochiasäure und Nephropathien etwa bei den Patienten in Frankreich (Prävalenz von 1\%). Wäre Aristolochiasäure wirklich alleine und in konsistenter Weise für eine Nephropathieprävalenz von nur schon $1 \%$ o unter den Behandelten verantwortlich, dann wäre ein Verbot von Mitteln, die diesen Stoff (in einer bestimmten Dosis) enthalten, sicherlich zu diskutieren.

$X i X i n$ ist wie gesagt eine wärmende Droge; sie wärmt das Innere, vertreibt Kälte und Wind, wärmt die Lunge, stillt Husten sowie Schmerzen und schiesst die Meridiane und die Nase frei. Es enthält 1000-mal weniger Aristolochiasäure als die in die Nephropathiefälle involvierten Aristolochia-Arten. Xi Xin wird aufgrund der seit 1000 Jahren bekannten, atemlähmenden Toxizität (heute den Safrolen angelastet) eher niedrig dosiert oder gut gekocht. Sie lässt aber nicht die Nieren erkalten wie die AristolochiaArten. So ist sie nie in die Nephropathiefälle involviert gewesen. Werden die Zusammenhänge so geordnet, dann ergibt es Sinn und wird konsistent: Aristolochiasäure in falsch und zu lange verwendeten kalten Drogen bei vorbestehender Schwäche des Nieren-Yang ist in die Entstehung von Nephropathien involviert, nicht aber der Gebrauch des wärmenden Xi Xin. Im Übrigen lässt sich auch die altbekannte, atemlähmende Toxizität der Droge $X i$ Xin gut als das Ergebnis von Effektmodifikationen erkennen. Beim ungeeigneten Patienten und in der falschen Dosierung ist es eher giftig, beim richtigen Patienten ist es hingegen heilsam. Schon vor fast 2000 Jahren hatte Zhang Zhong Jing im Shang Han Lun hohe Dosierungen von Xi Xin bei Kälteschädigungen beschrieben. Ohne Kältepathologie und ohne Yang-Mangel sind diese Dosierungen eher gefährlich. Der falsche Wirt bzw. die falsche Diagnose macht ein Heilmittel zu Gift und der richtige Wirt bzw. die richtige Diagnose macht ein Gift zum Heilmittel.

\section{Xi Xin (Asarum) in klassischen Rezepturen}

Die klassischen Rezepturen des Shang Han Lun haben einen taoistischen Hintergrund [12] und entsprechen einer Gesamtstrategie, die nicht einfach einer Addition der 
Wirkungen der Einzelsubstanzen entspricht. Steigen und Sinken sind dann nicht gegensätzliche Wirkungen, sondern beide unterstützen z.B. das Drehen im Energiekreislauf und verbinden oben mit unten. Erst die Kombination mit (blut-)kühlenden Mitteln erlaubt bei vielen Patienten ein kräftiges Wärmen und effektives Zerstreuen von Kälte, weil - metaphorisch gesprochen - das kühlende Öl dafür sorgt, dass der heiss laufende Motor nicht blockiert. Blutkühlende Mittel allein wären hingegen völlig sinnlos, und je nach Situation wären wärmende Mittel allein zu gefährlich. Die Rezeptstrategien neutralisieren gefährliche Nebenwirkungen eines Einzelmittels; die Wirkungen anderer Einzelmittel ergänzen sich. So werden gefährliche Mittel ungefährlich und so kann ein allein unwirksames Einzelmittel mithilfe eines anderen Mittels Wirkung entfalten.

Einige Rezeptstrategien, die $X i$ Xin enthalten, sind z.B. Danggui Sini Tang, Mahuang Fuzi Xixin Tang, Xiao Qinglong Tang und Dahuang Fuzi Xixin Tang.

- Danggui Sini Tang enthält Dang Gui, Gui Zhi, Bai Shao, Xi Xin, Gan Cao, Chuan Mu Tong und Da Zao. Es treibt Kälte aus dem Jueyin (6. Schicht), bewegt das Blut und schiesst die Meridiane frei. Oft ergänzen wir mit Fu Zi.

- Mahuang Fuzi Xixin Tang enthält Ma Huang, Fu Zi und Xi Xin, gut ergänzbar z.B. mit Gui Zhi, Gan Jiang, Gan Cao. Es zerstreut Kälte und Nässe aus dem Shao Yin (5. Schicht) und dem Tai Yang (1. Schicht). Ma Huang und Gui Zhi öffnen, sind warm und treiben Kälte aus, unterstützt durch $F u Z i$, das die innere Wärme zurückholt und das wahre Yang stärkt. Gan Jiang wärmt und Gan Cao harmonisiert die Mitte und verteilt die Wärme. Das durchschiessende Xi Xin löst den Schleim und unterstützt die Gesamtstrategie, die Kälte aus dem ganzen Körper vertreibt.

- Xiao Qinglong Tang enthält Ma Huang, Gui Zhi, Gan Jiang, Wu Wei Zi, Xi Xin, Ban Xia, Gan Cao und Bai Shao. Es ist dem Mahuang Fuzi Xixin Tang ähnlich, eliminiert Kälte und Nässe, wobei der Schwerpunkt aber auf dem Stillen des Hustens liegt. Xi Xin zerstreut Kälte und unterstützt Ban Xia im Lösen von Schleim. $W u$ Wei $Z i$ wirkt zurückziehend und damit schützend auf die Lunge. Bai Shao hält das Blut kühl und fliessend.

- Dahuang Fuzi Xixin Tang (aus dem Jin Kui Yao Lue) enthält $\mathrm{Da} H u a n g, F u \mathrm{Zi}$ und Xi Xin. Es wärmt den unteren Erwärmer und führt ab. Da Huang ist kalt und abführend, Fu $Z i$ wärmt unten, in der Mitte und oben, $X i$ Xin unterstützt $\mathrm{Da}$ Huang in der durchdringenden Wirkung, neutralisiert aber zusammen mit $F u ~ Z i$ die schädigende Kälte von $D a$ Huang. Eine sehr gute Strategie, die insgesamt die kühlende und damit darmlähmende Wirkung der westlichen Abführmittel nicht hat und deshalb die Darmkälte als sehr häufigen Grund der Verstopfung nicht chronifiziert, sondern behebt und eine gesunde, «warme» Peristaltik ermöglicht.

\section{Xi Xin (Asarum) in einem Fallbeispiel aus der Praxis}

Eine heute 75-jährige Patientin ist seit Sommer 2005 wegen einer Polymyalgia rheumatica in Behandlung mit chinesischen Kräutern, wobei sie seit 2008 nur noch im Winter Kräuter nimmt - im Winter ist der Körper geschlossen; das Yang sammelt sich dann am effektivsten. Die Schmerzen traten 2 Jahre nach einer vorangegangenen Herzoperation (Aortenklappenersatz) auf und verstärkten sich schubweise. Behandelt wurde bis anhin mit Steroiden $(20 \mathrm{mg}$ Prednisolon/Tag im Sommer 2004). Steroide sind günstiger als Basistherapeutika, weil letztere an die «Nieren» gehen und das Yang zu stark erschöpfen. Schon vor der Steroidtherapie bestanden Schlafstörungen und zweimal Nykturie. Am Mittag liessen die Schmerzen jeweils deutlich nach - mit dem Yang-Zenit. In der Anamnese zeigten sich zudem lumbale Schmerzen seit den Schwangerschaften und strengen Geburten, eine Hysterektomie im Alter von 50 Jahren wegen starker Blutungen, eine Cholezystektomie mit 60, drei Pneumonien in jungem Alter, prämenstruelles Syndrom/PMS, Reizdarm, schlecht schwitzen und nie richtig Fieber sowie kalte Füsse, wobei letztere Beschwerden mit zunehmendem Alter verschwanden (d.h. die Kälte hatte sich in Hitze gewandelt). Zunge: weiss belegt, vor allem auf Wurzel, gestaut «rot», dahinter blass, trocken, Schleimaspekt. Puls: Medikamentenpuls, dünn. Westliche Medikamente: Metoprolol, Lisinopril, Omeprazol, Kalzium- $\mathrm{D}_{3}$, Marcoumar.

Ein Bericht an den Hausarzt von Ende 2005 beschrieb den Fall so:

«Sehr geehrter Kollege, (...). Die wahrscheinliche Diagnose einer Polymyalgia rheumatica entspricht nach altchinesischer Sicht im Wesentlichen einer Kältekrankheit (...). Die Schlafstörungen entsprechen der gestörten 〈Herz〉-Funktion, die mangelnde 〈Nieren〉Wärme zeigt sich etwa an der Nykturie. Klinisch wesentlich ist, dass die Entzündungen als falsche Hitze begriffen werden, durch Kälte, die bremst und sich zu Hitze gewandelt hat, oder wie ein zu kleiner Motor (geschwächte «Nierenenergie`), der heiss gelaufen ist. Therapeutisch ist deshalb ein konsequentes inneres Wärmen mit entsprechenden Kräutern angesagt, und dadurch wird die Hitze weggehen. Die Patientin kann nach 2-3 Wintern wieder genügend innere Wärme aufbauen, um das Rheuma ganz wegzubringen. Die Polymyalgie ist aber nur ein Symptom, auch andere Symptome wie der Schlaf werden sich wieder verbessern, und die verlorene Energie kommt zurück. Dies ist der Grund, weshalb die Patientin die Kräuterbehandlung bereits jetzt als angenehm empfindet, obwohl die Beweglichkeit im Bewegungsapparat noch kaum besser geworden ist. Der bisherige Verlauf sieht sehr erfolgversprechend aus. Kortison ist bei diesem Bild ein potenter Kühler der falschen Hitze. Es ist in der Wirkung kalt und verstärkt deshalb tendenziell die Kälte, die ohnehin das Hauptproblem ist. Man könnte Kortison einsetzen, die Kräuter kämen auch mit dieser zusätzlichen Kälte mit der Zeit zurecht. Auch Diclofenac kühlt, nicht so potent und nicht so stark. Die Strategie besteht deshalb im Wärmen des ganzen Organismus und im Kühlen der falschen Hitze in Form der rheumatischen Entzündung nur im Notfall (dann am besten mit Prednisolon). Massgebend ist dabei der subjektive Leidensdruck; es macht nichts, wenn der CRP-Wert sich vorläufig noch nicht normalisiert oder sogar noch steigt. Die Patientin glaubt, dass sie im Moment sogar ohne Diclofenac auskommt. (...) Bitte machen Sie 
im Winter (November bis Anfang Februar) möglichst wenig Blutentnahmen, weil dadurch der Wärmeaufbau leidet (es läuft nicht ganz nach rein physikalischen Gesichtspunkten).»

Weil die ältere Patientin auch die Ernährung wieder konsequent auf die althergebrachte, wärmende Art ausrichtete und sie noch wusste, wie man früher auch in der Schweiz richtig gut gekocht hat, waren die Voraussetzungen günstig. Die altchinesische Rezeptstrategie bestand aus folgender Zusammensetzung, die gelegentlich leicht verändert und angepasst wurde - abgegeben als Granulat $3 \times 3-5 \mathrm{~g}$ täglich: 15 g Ma Huang, 54 g Fu Zi, 12 g Xi Xin, 40 g Gui Zhi, $30 \mathrm{~g}$ Gan Jiang, $30 \mathrm{~g}$ Gan Cao, $40 \mathrm{~g}$ Bai Zhu, $40 \mathrm{~g}$ Sheng Jiang, $20 \mathrm{~g}$ Dan Shen.

Unter der Supervision von Hin Chung Got (Meister der Altchinesischen Medizin, Schüler von Buqi Tang, dem Herausgeber von Zheng Qin-An's Schriften zur Feuerlehre [12]) wurde die Rezeptstrategie 2006 noch verbessert und Gan Jiang, Bai Zhu, Sheng Jiang und Dan Shen wurden mit Liang Jiang, Fu Ling, Ji Xue Teng und Yi Yi Ren ersetzt: $15 \mathrm{~g}$ Ma Huang, 63 g Fu Zi, 10 g Xi Xin, 40 g Gui Zhi, 40 g Liang Jiang, $30 \mathrm{~g}$ Gan Cao, $40 \mathrm{~g}$ Fu Ling, $30 \mathrm{~g}$ Ji Xue Teng, $60 \mathrm{~g}$ Yi Yi Ren. Fu Zi wurde später auf 90 g erhöht, Yi Yi Ren reduziert und die Rezeptstrategie in etwa so beibehalten.

Nach einigen Monaten der Behandlung waren Füsse und Hände kälter - ein Zeichen, dass die falsche Hitze am Schwinden war und die pathogene Kälte nun besser wahrgenommen wurde. Die Schmerzen wurden erträglich. Der vormals dünne Stuhlgang wurde geformter, der Schlaf besserte sich langsam und die Nykturie ging zurück - klare Zeichen, dass die innere Wärme sich sammelte. Allerdings gab es dabei Tage und Nächte mit starken Schmerzen («Aufräum»-Schmerzen), die die Patientin aber gut durchstehen konnte. Die Patientin nahm schon kurz nach Behandlungsbeginn kein Prednisolon mehr; gelegentlich brauchte sie in den ersten 6 Monaten noch Diclofenac. Der Hausarzt war beunruhigt wegen eines Blutdruckanstiegs und der erhöhten Entzündungsparameter, doch die Patientin liess sich beruhigen und nahm die Kräuter weiter. Der Blutdruckanstieg war vorübergehend. Ende 2006 bestanden Schmerzen nur noch in den Fingern (die wärmere Mitte treibt die Kälte in die Peripherie), kein Magenbrennen trat mehr auf, Wallungen und Nachtschweiss verschwanden. 2007 verschwanden auch die Restschmerzen und die Gelenksteifigkeit. Die Zunge wurde sauber, vitaler, weniger rot stagniert. Keine Nykturie mehr, Lebensfreude gut mit Gefühl von Stärke, und auch der vormalige Schwindel (bei Erschöpfung) verschwand fast ganz. Erkältungen wurden seltener, allerdings trat noch einmal eine Pneumonie auf, die jedoch gut überwunden wurde. Die Müdigkeit ist heute eher mehr als früher bei nun gutem Schlaf (Wärme macht müde; es ist eine gesunde Müdigkeit). Sehr gut wäre es, wenn wir die Behandlungen über die Winter weiterführen könnten, mit $X i$ Xin in der Rezeptstrategie!
So ungefähr verlaufen viele Fälle, bei denen YangSchwäche der Niere, der Milz/Mitte und von Herz und Lunge etwa zu entzündlich-rheumatischen Folgekrankheiten führen. Bei Jugendlichen ist das entzündliche Rheuma oft schon nach wenigen Wochen verschwunden, aber man sollte dann die Grundschwäche des Yang-Mangels über 1-2 Winter weiter behandeln, wobei immer eine gute, Yang-aufbauende Ernährung nötig ist. Xi Xin ist unentbehrlich in dieser Rezeptstrategie; es kann wie kein anderes Mittel die Nässe und Kälte aus allen drei Erwärmern und aus den Gelenken/Meridianen zerstreuen und so den ganzen Körper freimachen. Auch die Niere ist in diesem Fall mithilfe von Xi Xin energetisch stärker geworden, d.h. widerstandsfähiger gegen mögliche Nierenkrankheiten.

\section{Schlussfolgerungen}

Bei eingehender Betrachtung des Asarum-Verbotes durch die Swissmedic im Februar 2010 fällt auf, dass wir es zunehmend mit einer Wissenschaftskultur zu tun haben, die den Traum nach einer gefahrlosen Welt ins Masslose gesteigert hat, wobei schnell der Traum mit der Wirklichkeit verwechselt wird und die Dinge rasch in Gut und Böse, als kanzerogen, giftig oder «gesund» eingeteilt werden. Es erinnert an die berühmten Aussagen von Paracelsus in der Endphase der Scholastik vor 500 Jahren, von denen auch diese überliefert ist: «Kein Arzt oder Skribent hat nach rechtem Grund beschrieben, was in den Dingen der Natur sei. Alle haben sie nur nach dem Hörensagen geschrieben.»

Und es fällt auf, dass das Verbot des (bei richtigem Gebrauch) harmlosen Asarum mit vielen Medikamenten der Pharmaindustrie kontrastiert, deren kanzerogene Wirkungen aufgrund kontrollierter Studien tatsächlich dokumentiert sind und die dennoch zugelassen sind. Sinnvollerweise, denn wir erwarten vom Arzt, dass er Medikamente richtig anwendet - und dort, wo sie mehr nützen als schaden. Xi Xin (Asarum) muss als harmlos betrachtet werden. Dass es bei ganz falscher Anwendung beim falschen Patienten schaden könnte, potenziert durch weitere unsorgfältig verwendete westliche Mittel, würde ein Arzt der chinesischen Medizin ja befürchten müssen - aber wieso soll er es vorsätzlich schädigend verwenden?

Alles ist Gift oder Heilmittel - es kommt darauf an! Auch chinesische Kräuterdrogen können schädliche Wirkungen haben, wenn die Indikation falsch ist (und eine Rezeptur nicht gut ausbalanciert ist). Mit der Metapher vom Feuer lässt sich die grosse Bedeutung von Effektmodifikationen leicht erkennen, die in einem zu praxisfremden, modernen Sicherheitsverständnis wieder vergessen zu gehen droht: Ein kleines Zündholz ist brandgefährlich, wenn unser Patient ein Heustock ist. Ist unser Patient jedoch ein Eiszapfen, dann ist nicht einmal 
eine Fackel in der Lage, diesen zu entzünden oder zu sengen. Soll man wegen dem möglichen Zäuseln im Heustock das Feuer verbieten? Die Menschen haben das nie gemacht, weil das Feuer zu kostbar ist und weil wir Heu und Eis zu unterscheiden wissen. Man soll also den Ärzten der chinesischen Medizin, die zu unterscheiden wissen, nicht das kostbare Asarum verbieten. Und man soll es auch den Patienten, die es benötigen, nicht wegnehmen. Ein Verbot in der Schweiz entspricht zudem einer durch den «Volkswillen» obsolet gewordenen Benachteiligung komplementärmedizinischer Arzneien gegenüber den Arzneien der Pharmaindustrie. Dieser Vorwurf gründet auf unserer Auffassung, dass das Stimmvolk bei der Annahme der Initiative auch die akademischen Beurteilungskriterien zurückgewiesen hat, die Wirksamkeit und Nutzen der Komplementärmedizin in Abrede stellen wollten. Wir glauben, dass unsere Analyse zeigt, dass heute in der Tat falsche Beurteilungskriterien dominieren, auch wenn wir den Mangel an guter Nutzenerforschung in der Komplementärmedizin sehen. Das Asarum-Verbot beruht auf einer falschen Beurteilung und muss nach unserer Meinung wieder aufgehoben werden. Wir müssen das wichtige und unentbehrliche Mittel gezielt in den Fällen einsetzen können, wo es eine gute Wirkung hat und nützt.

\section{Disclosure Statement}

SB ist ein Teilhaber der Complemedis AG, die chinesische Arzneimittel vertreibt. Die Autoren haben keinerlei Art von Unterstützung für diesen Beitrag erhalten. Ein Interessenkonflikt besteht nicht.

\section{Literatur}

1 Bokemeyer C, Kollmannsberger C: Kanzerogenität; in Schmoll H-J, Höffken K (Hrsg): Kompendium Internistische Onkologie 2006. Berlin, Springer, 2006, pp 1972-1985.

2 Ghadimi BM, Kanger C, Becker H: Zum kanzerogenen Potential von Biomaterialien in der Hernienchirurgie. Chirurg 2002;73:833-837.

3 Rothman KJ: Epidemiology: An Introduction. New York, Oxford University Press, 2002.

4 Reginster F, Jadoul M, van Ypersele de Strihou C: Chinese herbs nephropathy presentation, natural history and fate after transplantation. Nephrol Dial Transplant 1997;12:81-86.

5 Nortier JL, Martinez MC, Schmeiser HH, Arlt VM, Bieler CA, Petein M, Depierreux MF, De Pauw L, Abramowicz D, Vereerstraeten P, Vanherweghem JL: Urothelial carcinoma associated with the use of a Chinese herb (Aristolochia fangchi). N Engl J Med 2000;342:1686-1692.

6 Wiebrecht A: Über die Aristolochia-Nephropathie. Dtsch Z Akupunktur 2000;3:187-197

7 Wiebrecht A: Obsolete Drogen in der chinesischen Medizin. Chin Med 2010;25:187-198

8 Zhao Z-Z, Liang Z-T, Jiang Z-H, Leung K-S, Chan C-L, Chan H-Y, Sin J, Man T-O, Law K-W: Comparative study on the aristolochic acid I content of Herba Asari for safe use. Phytomedicine 2008;15:741-748.

9 Zhongyao renmin gongheguo yaodian 中华人民共和国药典 (Pharmakopöe der Volksrepublik China, Ausgabe 2005).

10 Arlt VM, Stiborova M, Schmeiser HH: Aristolochic acid as a probable human cancer hazard in herbal remedies: a review. Mutagenesis 2002;17:265-277.

11 Chen T: Genotoxicity of aristolochic acid: a review. J Food Drug Anal 2007;15:387-399.

12 Neeb G: Vom Füllen der Leere - Yang: Das Feuer des Lebens. Schiedlberg, Bacopa, 2011 